\title{
Pulmonary artery sling anomaly presenting with unilateral lung hyperinflation: A case report
}

\author{
Hilal Şahin, Yeliz Pekçevik \\ Department of Radiology, Tepecik Training and Research Hospital, Izmir, Turkey. \\ E-mail: hilalcimen@gmail.com \\ Received: 30th January 2017, Revised: 10th March 2017, Accepted: 12th March 2017
}

\begin{abstract}
SUMMARY: Şahin H, Pekçevik Y. Pulmonary artery sling anomaly presenting with unilateral lung hyperinflation: A case report. Turk J Pediatr 2018; 60: 194-197.

Left pulmonary artery sling is a rare congenital pulmonary artery anomaly which presents with tracheal compression due to vascular ring formation. Rarely, patients may present with hyperinflation or collapse of a lung unilaterally. We present a one-month-old infant with dyspnea who had a hyperinflated left lung and collapsed right lung. Computed tomography (CT) angiography demonstrated pulmonary sling causing compression of proximal parts of right and left main bronchi. This case highlights the importance of three-dimensional CT angiography in evaluation of thoracic vascular anomalies associated with tracheobronchial pathologies.
\end{abstract}

Key words: pulmonary artery sling anomaly, tracheobronchial compression, hyperinflation, computed tomography angiography.

A pulmonary artery sling is a rare developmental vascular anomaly in which left pulmonary artery arises from the right pulmonary artery. As it passes between the trachea posteriorly and the esophagus anteriorly, it forms a sling around the trachea. ${ }^{1}$ A pulmonary sling is frequently associated with distal tracheal stenosis caused by congenital cartilaginous tracheal rings; which is then referred to as the ring-sling complex. ${ }^{2}$ In severe cases, the left pulmonary artery coursing around the distal trachea and the right main stem bronchus cause a ball-valve effect where the right lung becomes hyperinflated. ${ }^{3}$

Here, we report a one-month-old male infant with a pulmonary sling anomaly presenting with unilateral left lung hyperinflation.

\section{Case Report}

A one-month-old full term male infant, with birth weight of 2,700 grams, presented to emergency service with coughing, wheezing, dyspnea and tachypnea for three days. He had no history of fever, airway secretion or cyanosis. On physical examination, patient was a well-nourished baby with normal weight and length for his age. Respiratory rate was 50-60 breaths per minute. Auscultatory examination revealed prolonged expiration with normal respiratory sounds without additional breath sounds including crepitation. Heart sounds were normal and there was no abdominal mass or organomegaly. Laboratory studies including renal and liver function tests were in normal limits.

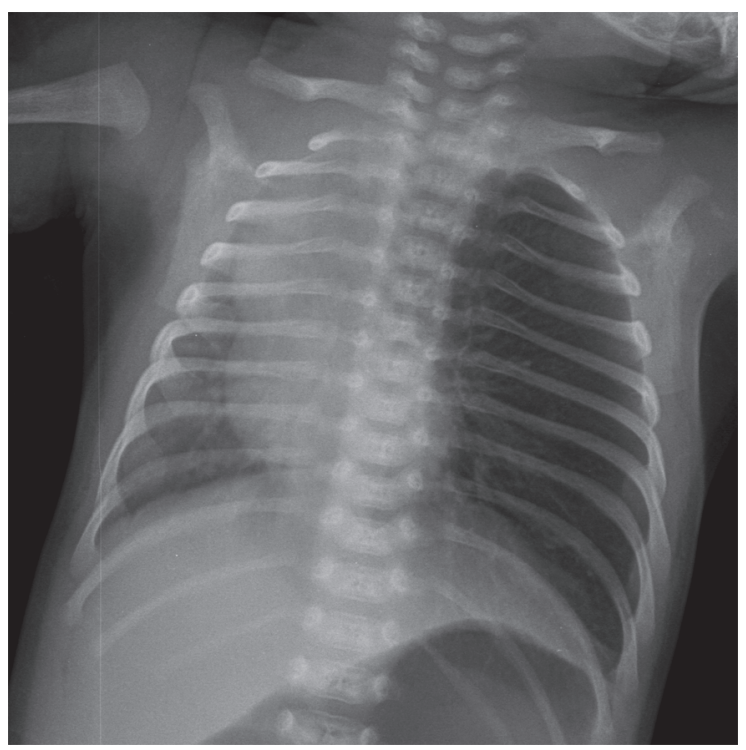

Fig 1. Anteroposterior chest X-ray shows that the mediastinum shifted to the right side and increased radiolucency of the left lung. 


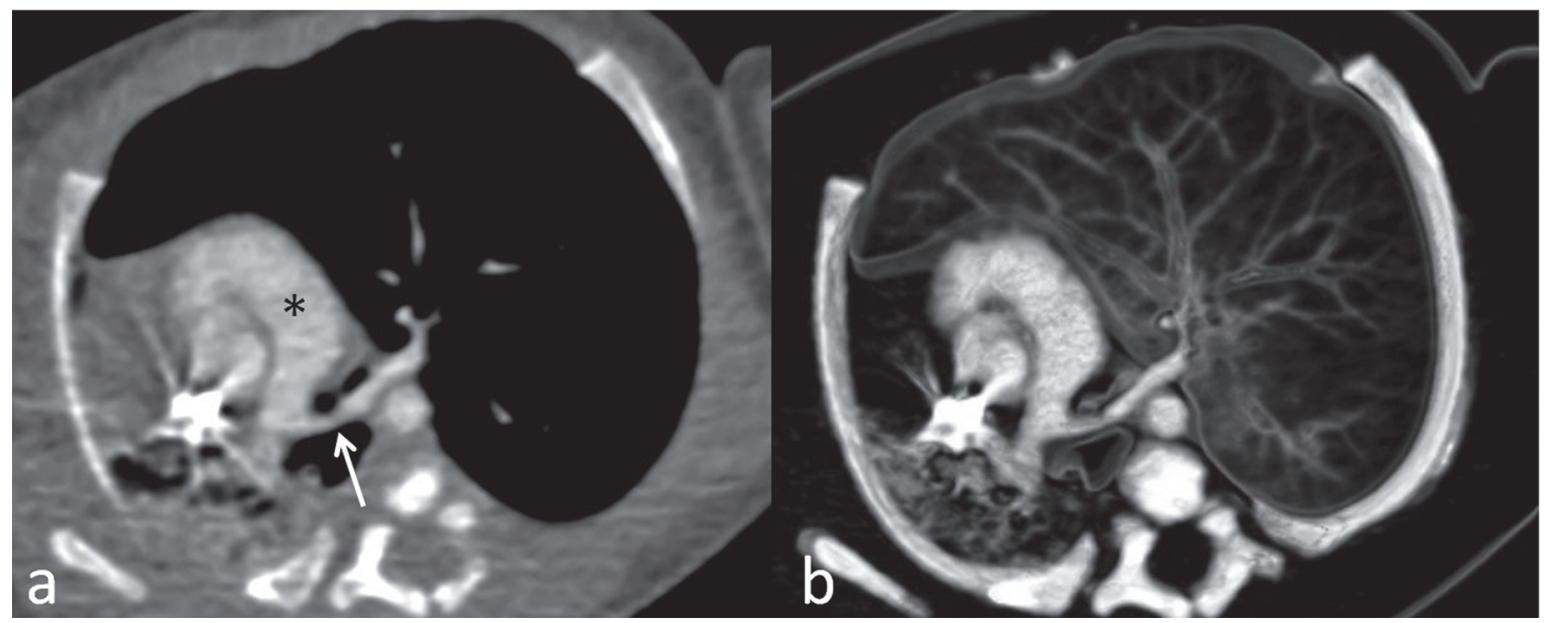

Fig 2. Axial oblique multiplanar reformatted image (a) and volume rendered image (b) show left pulmonary artery (LPA) (white arrow) arises from posterior wall of the proximal part of right pulmonary artery. Main pulmonary trunk (asterisk) is normal. Aberrant LPA courses posterior to both main stem bronchi and anterior to esophagus to reach the left hilum.

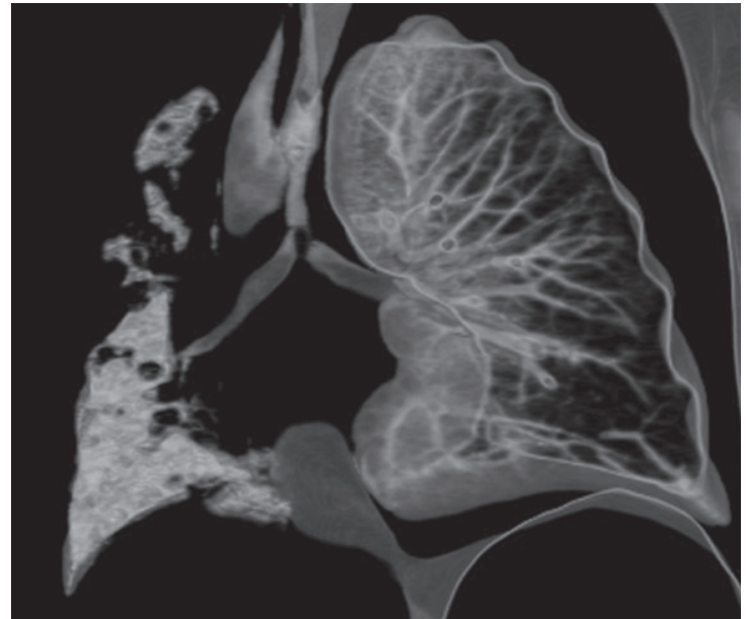

Fig 3. Volume rendered coronal image shows stenosis of the proximal parts of the main stem bronchi which is more severe on the left side. Note the dilated esophageal lumen with air on the right side of the trachea.

Chest X-ray demonstrated that mediastinum was shifted to the right with volume loss especially in right apical region and radiolucency of the left lung was increased due to hyperinflation (Fig. 1).

The patient was hospitalized and treated with high flow oxygen. However, symptoms did not regress in following 6 days and chest X-rays were similar.Bronchoscopy was performed and stenosis of left main bronchus with a normal trachea with pars membranacea was seen. Cardiac echocardiography revealed $6 \mathrm{~mm}$ atrial septal defect. Since the aortic arc was not evaluated properly, the patient underwent a computed tomography (CT) angiography examination. A left pulmonary artery (LPA) sling forming a vascular ring was demonstrated (Fig. 2) by CT angiography. The carina was low lying at T5-6 with compression of pulmonary sling to main stem bronchi just below the carina (Fig. 3). The proximal part of left main bronchus was severely collapsed with associated bronchomalacia. Left lung was hyperinflated and herniated to the right hemithorax. Right lung was collapsed as a result of stenosis of right main bronchi and compression of the left lung.

The patient received LPA re-implantation without tracheobronchial intervention with a slight residual stenosis in left main bronchus (Fig. 4). In the postoperative period, patient was followed in intensive care unit under mechanical ventilation due to intermittent desaturation episodes. After 19 days, he was extubated and taken to inpatient clinic for follow-up. In serial chest X-rays, the right lung started to inflate gradually with residual right mediastinal shift (Fig. 5). The patient was discharged after 10 days when symptoms of dyspnea regressed with normal blood gas levels and he was healthy in one-year follow-up period.

A written informed consent was obtained from the parents of the patient for this case report. 


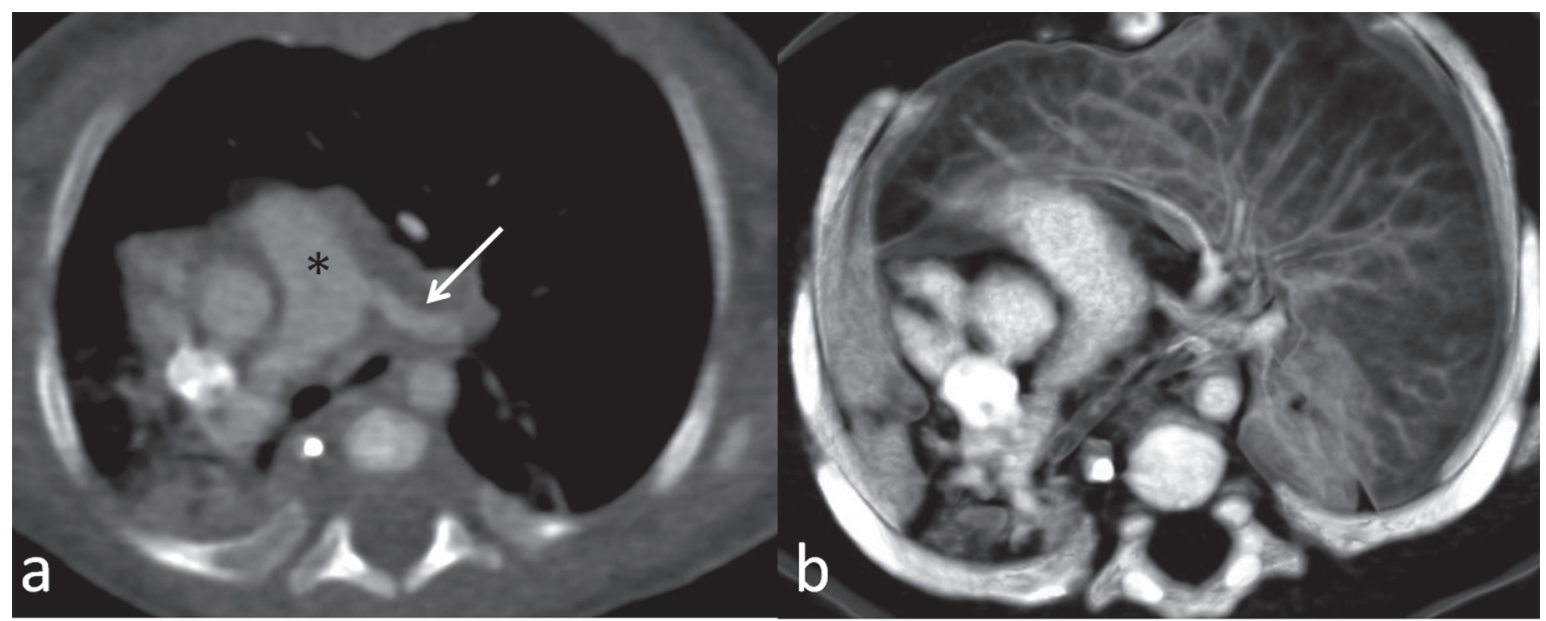

Fig 4. Axial oblique multiplanar reformatted image (a) and volume rendered image (b) shows main pulmonary trunk (asterisk) and re-implanted left pulmonary artery (white arrow). Note the residual stenosis of the left main bronchus and persisting hyperinflation of the left lung

\section{Discussion}

Respiratory stress is a frequent symptom in patients with congenital vascular anomalies. In LPA sling anomaly, respiratory distress occurs due to tracheobronchial compression by the aberrant left pulmonary artery as it passes over the right bronchus and goes posteriorly between the trachea and the esophagus and/ or associated tracheobronchial anomalies. ${ }^{4}$ The pulmonary artery sling anomaly is frequently associated with tracheobronchial abnormalities causing stenosis, tracheomalasia, and airway compression, as well as cardiac defects and non-cardiac lesions. ${ }^{1}$ Tracheobronchial anatomy and cardiovascular anomalies determine the morbidity and mortality, with the former being the most important prognostic factor. 4

In 1988, Wells et al. ${ }^{5}$ introduced a classification of pulmonary sling anomalies with two types mainly based on the anatomy of tracheobronchial tree (carina at T4-5 vs T5-6) and two subtypes according to the presence or absence of right upper lobe bronchus. According to Well's classification, the tracheobronchial anatomy was consistent with type 2 anatomy (low tracheal bifurcation) in our case. Besides, trachea was normal with a normal right upper lobe bronchus, consistent with the subtype B. Type 2 associations are more complex; therefore, they pose challenges in diagnosis and treatment, and require a multidisciplinary approach for preoperative planning and successful repair. ${ }^{6}$

In the pediatric patients, pulmonary sling anomaly is mostly characterized by symptoms related to compression of adjacent structures whereas it is usually an incidental finding in adults ${ }^{1}$. Tracheobronchial tree compression causes expiratory stridor, recurrent respiratory infections, and esophagus compression causes dysphagia and vomiting. Associated cardiovascular anomalies may cause other symptoms such as cyanosis. In imaging, usually short or long segment of tracheal stenosis is seen. ${ }^{7}$ However, solely bronchial abnormality is very rare as in the presented case. In a recent study by Chao et al., 8 funnel type tracheal stenosis was shown to accompany pulmonary sling in significantly higher rate than hypoplasia or segmental tracheal stenosis. Isolated bronchial stenosis with pulmonary sling was not reported

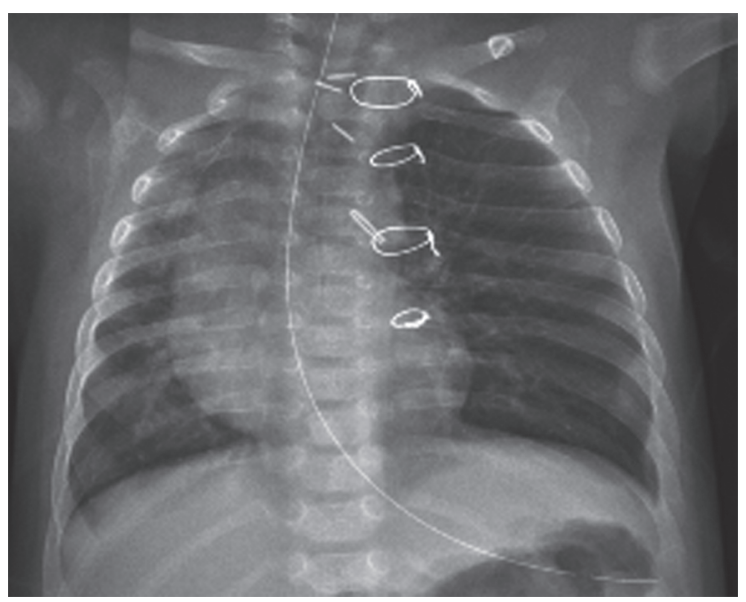

Fig. 5. Anteroposterior chest X-ray shows the increased inflation of the right lung and the residual mediastinal shift in the postoperative period. 
in their study. Pulmonary sling patients with isolated bronchial stenosis may present with ventilation abnormalities such as unilateral hyperinflation or collapse. Hyperinflation of a lung occurs as a consequence of "ball valve" effect in which air can flow into the lung distal to compressed segment on inspiration but cannot flow out of the lung on expiration. Unilateral hyperinflation of lung has a broad differential diagnosis including foreign body aspiration. ${ }^{9}$ Pulmonary sling is a rare cause of air trapping due to substantial bronchial narrowing and should not be forgotten in differential diagnosis of unilateral hyperlucent lung.

Evaluation of patients with suspected pulmonary sling should include echocardiography, CT imaging and bronchoscopy. ${ }^{3}$ Echocardiography is usually used as the first choice for diagnosis of pulmonary sling; however, the description of tracheobronchial tree anatomy is limited. Tracheobronchoscopy accurately identifies complete tracheal cartilages accompanying sling anomaly and provides texture and color details of the mucosa as well as information of dynamic changes of the airways. ${ }^{10}$ Although MRI has advantages in the evaluation of pulmonary sling such as avoiding ionizing radiation, it is time consuming and prolonged sedation is not appropriate for infants with severe respiratory distress. ${ }^{7}$ On the other hand, CT angiography has short examination time and exhibits better visualization of bronchial tree anatomy. High quality two- and three- dimensional multiplanar reformatted images or volumerendered images could delineate pathological morphological features of cardiovascular system or tracheobronchial tree accurately. ${ }^{7}$ Briefly, in all patients with pulmonary artery sling, CT angiography and tracheobronchoscopy are both necessary due to concomitant various tracheal anomalies.

In conclusion, in the differential diagnosis of unilateral hyperinflation, vascular anomalies should be kept in mind. Presence of tracheobronchial anomaly is the most important prognostic factor and plays a crucial role in the management of patients with pulmonary sling. $\mathrm{CT}$ angiography provides excellent images with multiplanar reformatting and three-dimensional volume rendering techniques in assessment of both vascular and tracheobronchial anomalies.

\section{REFERENCES}

1. Lee $\mathrm{KH}$, Yoon CS, Choe $\mathrm{KO}$, et al. Use of imaging for assessing anatomical relationships of tracheobronchial anomalies associated with left pulmonary artery sling. Pediatr Radiol 2001; 31: 269-278.

2. Berdon WE, Baker DH, Wung JT, et al. Complete cartilage-ring tracheal stenosis associated with anomalous left pulmonary artery: the ring-sling complex. Radiology 1984; 152: 57-64.

3. Backer CL, Mongé MC, Popescu AR, Eltayeb OM, Rastatter JC, Rigsby CK. Vascular rings. Semin Pediatr Surg 2016; 25: 165-175.

4. Zhong YM, Jaffe RB, Zhu M, Gao W, Sun AM, Wang Q. CT assessment of tracheobronchial anomaly in left pulmonary artery sling. Pediatr Radiol 2010; 40: 1755-1762.

5. Wells TR, Gwinn JL, Landing BH, Stanley P. Reconsideration of the anatomy of sling left pulmonary artery: The association of one form with bridging bronchus and imperforate anus. Anatomic and diagnostic aspects. J Pediatr Surg 1988; 23: 892-898.

6. Berdon WE, Muensterer OJ, Zong YM, Backer CL. The triad of bridging bronchus malformation associated with left pulmonary artery sling and narrowing of the airway: The legacy of Wells and Landing. Pediatr Radiol 2012; 42: 215-219.

7. Hu XH, Pa ME, Shen QL, Huang GY. Multi-detector computed tomography evaluation of tracheobronchial anomaly in pediatric patients with left pulmonary artery sling. Chin Med J (Engl) 2013;126: 2790-2792.

8. Chao YC, Peng CC, Lee KS, Lin SM, Chen MR. The association of congenital tracheobronchial stenosis and cardiovascular anomalies. Int J Pediatr Otorhinolaryngol 2016; 83: 1-6.

9. Dillman JR, Sanchez R, Ladino-Torres MF, Yarram SG, Strouse PJ, Lucaya J. Expanding upon the unilateral hyperlucent hemithorax in children. Radiographics 2011; 31: 723-741.

10. Leonardi B, Secinaro A, Cutrera R, et al. Imaging modalities in children with vascular ring and pulmonary sling. Pediatr Pulmonol 2015; 50: 781-788. 